\title{
CD 10: A Novel Stromal Marker in Pathogenesis and Prognosis of Invasive Breast Carcinoma
}

\author{
Devi Jawahar ${ }^{1}$, Muruganantham Arunagirinathan ${ }^{2 *}$ and Induja Murali ${ }^{1}$ \\ 'Department of Pathology, Government Vellore Medical College \& Hospital, Vellore. India \\ ${ }^{2}$ Department of Pathology, Government Villupuram Medical College \& Hospital, Villupuram. India
}

\section{ABSTRACT}

Background: Breast carcinoma is the major cause of mortality and morbidity in Indian women. Though breast carcinoma arises from epithelial cells, stroma also plays a very important role in the pathogenesis of carcinoma breast which is assessed by the stromal marker CD 10.

Aim: To study the stromal expression of CD 10 in carcinoma breast and find its relationship with other prognostic markers and study the role of stroma in pathogenesis of breast carcinoma.

Materials and Methods: 50 cases of breast cancer were included in the study. H \& E slides were retrieved, reviewed and IHC was done with a panel of markers which included ER, PR, HER2/neu and CD 10. Stromal expression of CD 10 in invasive breast carcinoma was noted and analysed in correlation with other prognostic markers.

Results: Significances in the stromal CD 10 expression was calculated in regard to ER, PR \& HER2/neu. Higher the tumour grade, more was the CD 10 stromal expression.

Conclusion: As per the study, it is mandatory to include CD 10 stromal marker along with the panel of epithelial markers in invasive breast carcinoma. CD 10 contributes a potential target for development of novel therapies.

Keywords: CD 10 Stromal Marker, Invasive Breast Carcinoma, Immunohistochemistry.

\section{Introduction}

Breast cancer is one of the leading causes of morbidity and mortality among women in the developing and developed countries. According to the population based national cancer registry (India), it is one of the major cancers in Indian women. Though breast carcinoma is an epithelial malignancy, stroma plays a key role in its development and pathogenesis. ${ }^{[1]}$

Along with the epithelial immunohistochemical markers, stromal markers are now emerging as novel markers in assessing the prognosis of invasive breast cancer and is under trial. Along with the traditional prognostic factors like tumour staging, lymph node status and tumour grade, epithelial markers like estrogen receptor, progesterone receptor and HER2/neu receptor expression has emerged as predictors.

The newly emerging stromal marker is CD10, also called Neprilysin and Common Acute Lymphoblastic Leukemia/ Lymphoma Antigen (CALLA) which is a zinc dependent matrix Metalloproteinase. ${ }^{[2]}$ Physiologically CD10 is expressed on the epithelial cells of breast. In infiltrating carcinoma of the breast, CD10 positivity of stromal cells increases and positivity in epithelial cells decreases.
Recent studies show a strong correlation between stromal expression of CD10 and stage of the disease. CD10 positivity of stromal cells correlates with worse prognosis.

The newly emerging stromal marker CD10 is a zinc dependent metalloproteinase. Physiologically CD 10 is expressed on the epithelial cells of the breast. In infiltrating carcinoma of breast, CD10 positivity of stromal cells increases and positivity in epithelial cells decrease. CD10 positivity of stromal cells correlates with worse prognosis. The current study justifies the CD10 stromal marker in breast carcinoma for prognostication and therapy in invasive breast carcinoma. According to Makretsov NA et al studies ${ }^{[3]}$ stromal CD10 expression in invasive breast carcinoma showed statistically significant positive correlation with high tumour grade. There was a strong correlation between CD10 staining and ER negative status of the tumour. There was no correlation between stromal CD10 expression and lymph node status, tumour size, histological subtype, PR and HER2/neu status of the tumour. In Iwaya $\mathrm{K}$ et al studies, ${ }^{[4]} \mathrm{CD} 10$ was positive in $18 \%$ of invasive ductal carcinomas. No stromal expression of CD10 was detected in the normal breast or in 16 intra ductal carcinomas. The non neoplastic myoepithelial cells constantly expressed CD10 and has been used as a built- in 
positive control in this study. There was no expression of CD10 in ductal cells, fibroblasts and adipocytes. Masaki et $\mathrm{al}^{[5]}$ proposed that CD10 expression could be considered positive when more than $10 \%$ of the stromal cells in vicinity of the neoplastic cells were positive. Based on this criteria, they detected stromal CD10 expression in 19\% of IDC. Sayantan H Jana et.a[ ${ }^{[1]}$ studies concluded that stromal expression of CD10 in breast cancer correlates with high tumour grade, poor prognosis, ER negativity and HER2/neu positivity. Ali Taghizadeh et $\mathrm{al}^{[2]}$ studies detected stromal CD10 in $28 \%$ of IDC. This stromal CD10 expression in IDC has significantly correlated with increasing tumour size, increasing histologic grade, the presence of nodal metastases and estrogen receptor negative status.

\section{Aims and Objectives}

The aim of the present study is to evaluate the CD 10 stromal expression in breast carcinoma, to find its relationship with the other prognostic markers and study the role of stroma in breast cancer pathogenesis. Also included in the present study are the epithelial immunohistochemical markers such as estrogen receptor, progesterone receptor and HER2/neu receptor, which will be correlated with stromal marker CD 10.

\section{Materials and Methods}

This retrospective case series study was done in Department of Pathology, Government Vellore Medical College \& Hospital, Vellore, Tamil Nadu, India. 50 cases of breast carcinoma which were reported in the year 2018 were selected for evaluation. Hematoxylin and eosin stained slides were retrieved and reviewed. Immunohistochemistry was performed on the newly cut thin sections from corresponding paraffin blocks of the above mentioned 50 cases. The panel of IHC markers included were ER, PR, HER2/neu and CD10. Patients records from the reported data were collected and reviewed along with the slides. CD 10 positivity was analysed and correlated with the Estrogen receptor, Progesterone receptor and HER2/neu status.

\section{Outcomes of the study}

- The primary outcome of the study evaluates the CD 10 positivity in stromal cells of invasive breast carcinoma.

- The secondary outcome correlates and finds the relationship between the stromal marker CD10 and the expression of epithelial markers such as Estrogen Receptor, Progesterone Receptor and HER2/neu.

\section{Results}

ER, PR and HER2/ neu expression was evaluated according to the CAP protocols. ${ }^{[6]} \mathrm{ER}$ and PR are nuclear stains and HER2/neu is a cell membrane stain. CD10 was considered positive if more than $10 \%$ of the stromal cells showed cytoplasmic and cell membranous positivity. (Figure 1\&2).

A total of 50 breast carcinoma cases were included in the study. The age group of the Invasive ductal carcinoma(IDC) patients were between 21-92 years.( TABLE 1).

Most of the breast carcinoma patients were between 51-60 years of age(Table 1).

Out of the 50 cases of breast carcinoma in the study, 22 cases were CD10 positive, which is about $44 \%$ of the sample size.(Table 2).

Out of the 22 CD10 positive cases, 18 cases were Grade 2 / Grade 3 Invasive ductal carcinoma with high mitotic rate. 4 cases were Grade 1.(TABLE 3).

All the patients with CD10 positivity were negative for both ER \& PR and 12 patients who were CD10 positive showed positivity for HER2/neu. (Table 4).

The Fischer exact test ${ }^{[7]}$ statistical value was calculated to be $<0.00001$ for association of CD 10 with both ER and PR, which is statistically significant at $p<0.05$. The correlation between CD10 and HER2/neu was statistically significant, (i.e)Chi square value 36.33 was obtained in which $\mathrm{p}$ value is less than 0.001 .

Table 1 :Age group of patients with invasive ductal carcinoma breast in the present study.

\begin{tabular}{|c|c|c|}
\hline Age Group(Years) & Frequency & \% of sample size \\
\hline $21-30$ & 2 & 4 \\
\hline $31-40$ & 5 & 10 \\
\hline $41-50$ & 9 & 50 \\
\hline $51-60$ & 25 & 16 \\
\hline $61-70$ & 8 & - \\
\hline $71-80$ & - & - \\
\hline $81-90$ & - & 2 \\
\hline$>90$ & 1 & $100 \%$ \\
\hline Total & $\mathbf{5 0}$ & \\
\hline
\end{tabular}


Table 2: Age wise distribution of CD10 positivity.

\begin{tabular}{|c|c|c|}
\hline Age Group(Years) & No.of CD 10 Positive cases & \% of sample size \\
\hline $21-30$ & 1 & 4.5 \\
\hline $31-40$ & 3 & 13.5 \\
\hline $41-50$ & 4 & 50 \\
\hline $51-60$ & 11 & 9 \\
\hline $61-70$ & 2 & - \\
\hline $71-80$ & - & - \\
\hline $81-90$ & - & 4.5 \\
\hline$>90$ & 1 & 40 \\
\hline
\end{tabular}

Table 3: Association of CD 10 with tumour grade.

\begin{tabular}{|c|c|c|}
\hline Invasive ductal carcinoma & CD 10 + & CD 10 - \\
\hline Grade 1 & 4 & 10 \\
\hline Grade 2/ Grade 3 & 18 & 18 \\
\hline
\end{tabular}

Table 4: Association of CD 10 with ER, PR \& HER2/neu.

\begin{tabular}{|c|c|c|}
\hline & CD 10+ CD 10- \\
\hline ER + & Nil & 25 \\
\hline ER - & 22 & 3 \\
\hline PR + & Nil & 23 \\
\hline PR - & 22 & 5 \\
\hline HER2/neu + & 12 & 6 \\
\hline HER2/neu - & 10 & 22 \\
\hline
\end{tabular}

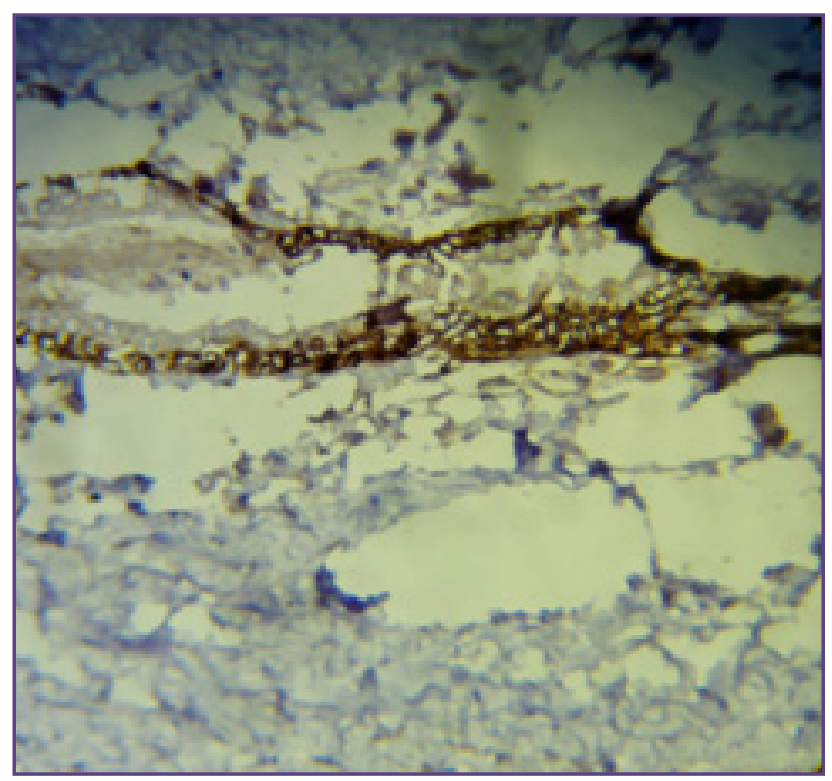

Fig. 1: CD 10 expression in myoepithelial cells of normal breast.

www.pacificejournals.com/apalm

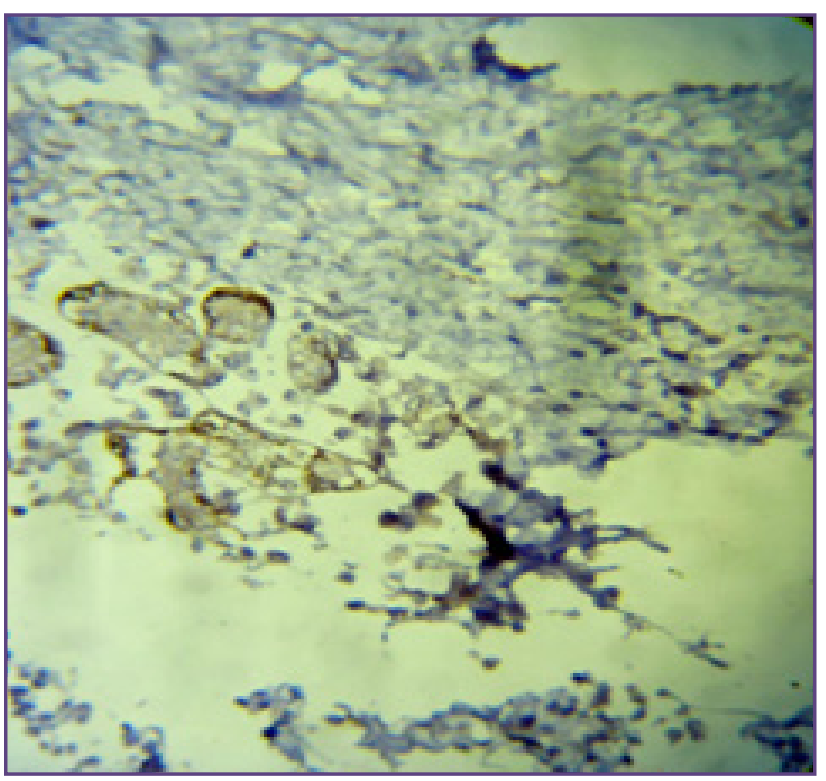

Fig. 2: Stromal CD 10 positivity in invasive ductal carcinoma.

eISSN: 2349-6983; pISSN: 2394-6466 


\section{Discussion}

CD 10 is a membrane bound zinc dependent endopeptidase (a type of MMP) which regulates the physiological action of various peptides by lowering their extracellular concentration available for receptor binding. ${ }^{[8,9]} \mathrm{CD} 10$ protease maintains the early progenitor population in the human mammary lineage by cleaving signalling proteins that promote differentiation of ECP s (early common progenitors) to LEPPs (luminal epithelial progenitor) or MEPPs (myoepithelial progenitor), which ultimately gives rise to luminal and myoepithelial cells respectively. Hence CD10 by its enzymatic functions (along with the help of beta integrin) acts largely as a Stem cell (SC) regulator in the breast, preventing the unchecked proliferation of mammary stem cells. ${ }^{[9,10,11]}$

In breast carcinoma, disappearance of $\mathrm{CD} 10$ positive myoepithelial cells and basement membrane leads to the progression of DCIS to invasive carcinoma \& CD10 expression of the stromal cells is correlated with poor prognosis, estrogen receptor negativity and high tumour grade.${ }^{[3,4,12,13,14]}$ According to Maguer -Satta et al, ${ }^{[8]}$ an early oncogenic event in stem cells modulate the expression of CD10 enzyme in the altered cells or even in the neighbouring cellular environment. A resultant decrease in CD10 enzymatic function after the neoplastic transformation of early common progenitors $(\mathrm{ECP})$ or progenitors $(\mathrm{P})$ could induce an accumulation of unprocessed peptides in the $\mathrm{SC}($ stem cell) microenvironment, resulting in their alteration of lineage commitment and malignant proliferation. ${ }^{[8,15]}$ This is the basis for progression of DCIS into invasive malignancy with CD10 loss.

Stromal expression of CD 10, a novel stromal marker was found to be significantly associated with high grade tumour, poor prognosis, increasing mitotic rate, ER negativity and HER2/neu positivity. CD10 constitutes a clinically important prognostic marker and potential target for development of novel therapies. This study will contribute to gain knowledge about the role of stroma and its marker CD10 in invasive breast cancer and its correlation with Estrogen receptor, Progesterone receptor and HER2/neu status and also aids in the role of stroma in breast cancer pathogenesis and Immunohistochemistry protocols.

Depending on the statistical correlation of CD 10 stromal marker with the epithelial Immunohistochemistry markers, our study will enable us to decide whether CD 10 should be included in the immunohistochemistry marker panel of Carcinoma breast. In this study, CD10 positivity in stromal cells is expressed in $44 \%$ of the sample size. Also in this study the correlation between CD10 and HER2/ neu is statistically significant and closely associated with one another (i.e)Chi square value 36.33 was obtained in which $\mathrm{p}$ value is less than 0.001 . This signifies the strong correlation between CD10 and HER2/neu. Thus CD 10 stromal expression and its close correlation with HER2/ neu is associated with poor prognosis. As per the present study there is a strong association found between ER and CD10 and also PR and CD10 according to Fisher exact test. For ER and CD10, the Fisher exact test ${ }^{[7]}$ gives the value of $<0.0001$ which is statistically significant. The Fisher exact test $^{\left[{ }^{7]}\right.}$ for PR and CD10 gives the value of $<0.0001$ which is also statistically significant.

\section{Conclusion}

Stroma plays a vital role in the development, progression, hormonal expression and response to chemotherapy in invasive ductal carcinoma of breast. CD10 may prove to be a potential target for new Oncotherapy, as it is associated with the cleavage of doxorubicin and leads to Chemo resistance. The expression of CD10 in the stroma of IDC is associated with ER negativity, HER2/neu positivity, higher tumour grade and poor prognosis. CD10 contributes a potential target for development of novel therapies.

\section{Summary}

The present study explains the role of stroma and CD 10 in invasive breast carcinoma prognosis. This study has established the significant correlation between CD 10 positivity and ER, PR and HER2/neu status. For majority of the patients who attend the Tertiary care centres with invasive breast carcinoma, this study will be useful in deciding the prognosis and further treatment protocol.

\section{References}

1. Sayantan HJ et al. CD 10-A new prognostic stromal marker in breast carcinoma, its utility, limitations and role in breast cancer pathogenesis. Indian Journal of Pathology and Microbiology 2014; October-December 57(4): 530-536.

2. Ali Taghizadeh - Kermani et al. The stromal overexpression of CD10 in Invasive breast Cancer and its association with clinicopathologic factors. Iranian journal of Cancer Prevention 2014; 7(1): 17-21.

3. Makretsov NA, Hayes M, Carter BA, Dabiri S, Gilks CB, Huntsman DG. Stromal CD10 expression in invasive breast carcinoma correlates with poor prognosis, estrogen receptor negatvity, and higher grade. Mod Pathol 2007; 20: 84-9.

4. Iwaya K, Ogawa H, Izumi M, Kuroda M, Mukai K. Stromal expression of CD10 in invasive breast carcinoma: A new predictor of clinical outcome. Virchows Arch 2002;440:58993.

5. Masaki T, Keiichi I, Masahiko K, Miki I. The stromal expression of CD10 in breast carcinoma. J of Tokyo Med University 2001;59: 45-50.

6. College of American pathologists[certified]: Protocol for the examination of specimens from patients with invasive 
carcinoma of the breast[Protocol web posting date: June 2012]. Available from: http://www.cap.org/apps/cap. portal-144 .

7. https://www.socscistatistics.com/tests/fisher/Default2.aspx .

8. Mageur-Sata V, Besancon R, Bacherlard- Cascales E. Concise review: Neutral endopeptidase (CD10): A multifaceted environment actor in stem cells, physiological mechanisms and cancer. Stem Cells 2011; 29: 389-96

9. Bacherlard- Cascales E, Chapellier M Delay E, Pochon G, Voeltzel T, Puisieux A et al. The CD10 enzyme is a key player to identify and regulate human mammary cells. Stem Cells 2010; 28: 1081-8.

10. Weil M, Itin A, Keshet E. A role for mesenchyme derived tachykinins in tooth and mammary gland morphogenesis. Development 1995;121:2419-28.

11. Kenny AJ, O' Hare MJ, Gusterson BA. Cell surface peptidases as modulators of growth and differentiation. Lancet $1989 ; 2: 785-7$.
12. Smollich M, Gote M, Yip GW Yong ES, Kersting $\mathrm{C}$,Fischgrabe $\mathrm{J}$ et al. On the role of endothelin-converting enzyme-1(ECE-1) and neprilysin in human breast cancer. Breast Cancer Res Treat 2007; 106:361-9.

13. Kesse-Adu R, Shousha S. Myoepithelial markers are expressed in at least $29 \%$ of oestrogen receptor negative invasive breast carcinoma. Mod Pathol 2004; 17:646-52.

14. Ashish Nitin Dhande et al. Stromal expression of CD 10 in breast carcinoma and its correlation with clinicopathological parameters. South Asian Journal of Cancer. 2019;8(1): 18-21.

15. Tahani Louhichi et al. Stromal CD 10 expression in breast cancer correlates with tumour invasion and cancer stem cell phenotype. BMC Cancer. 2018; 18: 49.

16. Thomas S. Babu RJ, Agarwal K, Pari V, Jain M, Andley M et al. Effect of neoadjuvant chemotherapy on stromal CD 10 antigen in breast cancer - a preliminary study. Indian $\mathrm{J}$ cancer 2013;50:46-51.

*Corresponding author:

Dr. A. Muruganantham, S/o N.K. Arunagirinathan., 42/1 C.Samudi Street., Nattarampalli., Tirupattur District., Tamil Nadu., India. Pin: 635852

Email: drmuruganantham@yahoo.in

Financial or other Competing Interests: None. 